\title{
Characterization of the development of Haemonchus contortus ZJ strain from gerbils
}

\author{
Yi Yang ${ }^{1}$, Xiaolu Guo ${ }^{1}$, Hongli Zhang ${ }^{2}$, Yan Huang ${ }^{1}$, Xueqiu Chen ${ }^{1}$ and Aifang Du ${ }^{1 *}$
}

\begin{abstract}
Background: Haemonchus contortus is a serious parasitic nematode in domestic ruminants around the world, including China. Haemonchus contortus has developed extensive resistance to commercial anthelmintics, which has produced a demand for new control methods, such as more effective drugs. Gerbils infected with $\mathrm{H}$. contortus have previously been used as a model for anthelmintics selections, and the growth of $H$. contortus had been briefly examined. To enhance the model, this study provides an additional description of the development of $\mathrm{H}$. contortus ZJ strain in gerbils.

Results: Gerbils were infected with H. contortus ZJ strain at a dose of 2000 exsheathed infective larvae (xL3s) and sacrificed at 4, 7 and 18 days post-infection (dpi). Only fourth-stage larvae were found in the stomachs. About 2\% of the inoculums were obtained at each of the three sampling time points. Larvae grew more slowly in gerbils than in sheep, but presented almost the same morphology. Rod-like crystalline inclusions were present in the intestinal cells of larvae, indicating that the metabolic rate of larvae was probably greatly reduced. Histological examination of stomach sections showed that larvae are located in the lumens or at the mucosal surfaces, with few inflammatory changes evident.

Conclusions: The development and features of H. contortus ZJ strain in gerbils were described. Our results provide supplementary information of $\mathrm{H}$. contortus growth in gerbils, especially the presence of rod-like crystalline inclusions, and may contribute to improve the anthelmintic selection system.
\end{abstract}

Keywords: Haemonchus contortus ZJ strain, Gerbil, Development, Rod-like crystalline inclusions

\section{Background}

The blood-feeding gastric nematode Haemonchus contortus is a serious pathogen in ruminants all around the world, including China. Drug resistance and lack of effective vaccines bring difficulties to the control of the disease. Previous studies have screened potential anthelmintics utilizing a $H$. contortus-infected gerbil model.

Conder et al. [1] first described the model for studying anthelmintics against $H$. contortus, and examined the growth and development of $H$. contortus in gerbils [2]; this study found that artificially exsheathed larvae were able to grow and establish successfully in gerbil's

\footnotetext{
* Correspondence: afdu@zju.edu.cn

${ }^{1}$ Institute of Preventive Veterinary Medicine, Zhejiang Provincial Key Laboratory of Preventive Veterinary Medicine, College of Animal Sciences, Zhejiang University, Hangzhou 310058, China

Full list of author information is available at the end of the article
}

stomach. However as compared to those in sheep, the larvae in gerbils exhibited slower and incomplete growth. De Jesus-Gabino et al. [3] and Suires et al. [4, 5] also tested the anthelmintic effects of different plant extracts on $H$. contortus in gerbils. In addition, other laboratory animals, such as mice, have been utilized to set up laboratory screening systems for anthelmintic activity against $H$. contortus [6, 7].

The slower and incomplete growth of $H$. contortus in gerbils was also observed as described in other laboratory animals. Hutchinson \& Slocombe [8] found that $H$. contortus completed its development at a slower rate than in sheep when they infected laboratory rabbits with exsheathed larvae. Even though a few female worms were pregnant, a high proportion of early fourth-stage larvae were recovered and some of them contained rod-like crystalline inclusions. Wagland et al. [9] demonstrated that $H$. 
contortus stayed and developed into fourth-stage in guinea pigs. Mature females, earlier stage larvae and fertile eggs were found in Chinchilla [10].

Usually, sheep were chosen as a suitable host for $H$. contortus research [11], but this procedure is expensive and time-consuming. A convenient and cost effective in vivo gerbil model is urgently needed. To improve the model, this experiment details the developmental morphology of $H$. contortus ZJ strain in gerbils and provides additional description of the growth.

\section{Methods}

\section{Gerbils and parasites}

Gerbils (Meriones unguiculatus) were purchased from Zhejiang Experimental Animal Center (certificate no. scxk (Zhe) 2014-0001). The eggs of Haemonchus contortus ZJ strain were collected from naturally singly-infected sheep. Infective larvae (iL3s) were cultured from eggs for 7 days at $28{ }^{\circ} \mathrm{C}$.

Haemonchus contortus exsheathment and gerbil infection The iL3s were exsheathed to xL3s using $0.2 \% \mathrm{NaOCl}$ based on the method described by Rothwell \& Sangster [12]. Thirty-three 6-month old male gerbils were selected, with body weight of $80 \mathrm{~g}$ each, and divided randomly into five groups: group $1(n=3)$, represented the control group with no treatment; group $2(n=3)$, the immunedepressed; group $3(n=9)$, the immune-depressed and infected, necropsy at $4 \mathrm{dpi}$; group $4(n=9)$, the immunedepressed and infected, necropsy at $7 \mathrm{dpi}$ and group 5 $(n=9)$, the immune-depressed and infected, necropsy at 18 dpi. Groups 2-5 were immune-depressed by intramuscular injection with dexamethasone sodium phosphate (1 ml:5 mg, Shenniao, Bailing Animal Medicine Co., Ltd., Ganzhou, China) at a dose of $0.5 \mathrm{mg} / \mathrm{kg}$ for two consecutive days. Groups 3-5 were orally infected with 2000 xL3s.

\section{Necropsy of gerbils}

All gerbils were euthanized and necropsy was carried out to obtain the stomachs. Six of the nine stomachs from groups 3-5 were used for larvae collection. Stomachs were digested in peptic- $\mathrm{HCl}$ and washed by phosphate-buffered saline (PBS) [11]. The number of larvae was counted with an anatomical lens (Motic, Fujian, China). Larvae were fixed in $7 \%$ formol saline and lengths $(n=10)$ were measured with a confocal laser scanning microscope (Olympus, Tokyo, Japan). Stomachs from groups $1-2$, and three of the nine stomachs from groups 3-5, were directly fixed in $10 \%$ neutral buffered formalin for histological study.

\section{Histological examination of stomach}

Each of the fixed stomachs was embedded in paraffin, mounted on glass sides and stained with hematoxylin- eosin (HE). Stained tissue sections were examined with an optical microscope (Olympus, Japan).

\section{Statistical analyses}

The number and length of larvae were shown as means \pm SD. A one-way ANOVA was conducted for length comparison between different groups.

\section{Results}

Larvae were able to colonize gerbil stomachs, however, only fourth-stage larvae were found. The amount of surviving larvae was $38.7 \pm 2.2,45.0 \pm 3.9$ and $41.3 \pm 2.8$ at 4 , 7 and $18 \mathrm{dpi}$, respectively (Table 1 ), which was $2 \%$ of the inoculums. The body lengths of larvae at the three sampling time points were $1100 \pm 169 \mu \mathrm{m}, 1273 \pm 122 \mu \mathrm{m}$ and $1553 \pm 133 \mu \mathrm{m}$, respectively (Table 1 ). There was a significant difference between larvae recovered at 4 and 18 dpi (ANOVA: $F_{(2,9)}=5.221, P=0.031$ ). This suggests larvae grew slowly across infection time.

Larvae recovered at 4 dpi displayed obvious sexual differentiation and could be identified as males and females. A swelling containing the vestigial copulatory bursa with short spicules was found at the posterior extremity of male larvae (Fig. 1a), but just a tail in the females (Fig. 1b). The well-developed excretory pore (Fig. 1c) and buccal capsule (Fig. 1d) were very prominent at $4 \mathrm{dpi}$, as was the genital primordium (Fig. 1e). Some differentiation into functional entities, such as the vulva ovejector primordium, had occurred in the genital primordium at $7 \mathrm{dpi}$ (Fig. 1f). One confusing finding was the appearance of a mass of strange tissues below the cuticle. The tissues could be seen in Fig.1b-f. Most of them were helically pipeshaped, and some were vesicle-shaped. The exact role of the specific subcutaneous substance was unknown and warrants follow-up studies.

The rod-like crystalline inclusions were present in $H$. contortus after $7 \mathrm{dpi}$ (Fig. 2) and could be found in almost all of nematodes. The crystals were located in the intestinal cells of larvae and were fairly uniform in their rod-like shape, but varied considerably in their sizes. In some cases, two seemingly identical crystals were found parallel to each other with a narrow space. Moreover, a small number of crystals were able to split in two, giving the appearance of a Y shape. The number of crystal was

Table 1 The number and length (mean \pm SD) of Haemonchus contortus larvae recovered in gerbils at necropsy on 4, 7 and 18 days post-infection

\begin{tabular}{llll}
\hline & \multicolumn{3}{l}{ Days post-infection } \\
\cline { 2 - 4 } & 4 & 7 & 18 \\
\hline Number & $38.7 \pm 2.2$ & $45.0 \pm 3.9$ & $41.3 \pm 2.8$ \\
Length $(\mu \mathrm{m})$ & $1100 \pm 169^{\mathrm{b}}$ & $1273 \pm 122^{\mathrm{ab}}$ & $1553 \pm 133^{\mathrm{a}}$ \\
\hline Note: ${ }^{\mathrm{a}}$ vs $^{\mathrm{b}}, \boldsymbol{P}<0.05$ & &
\end{tabular}



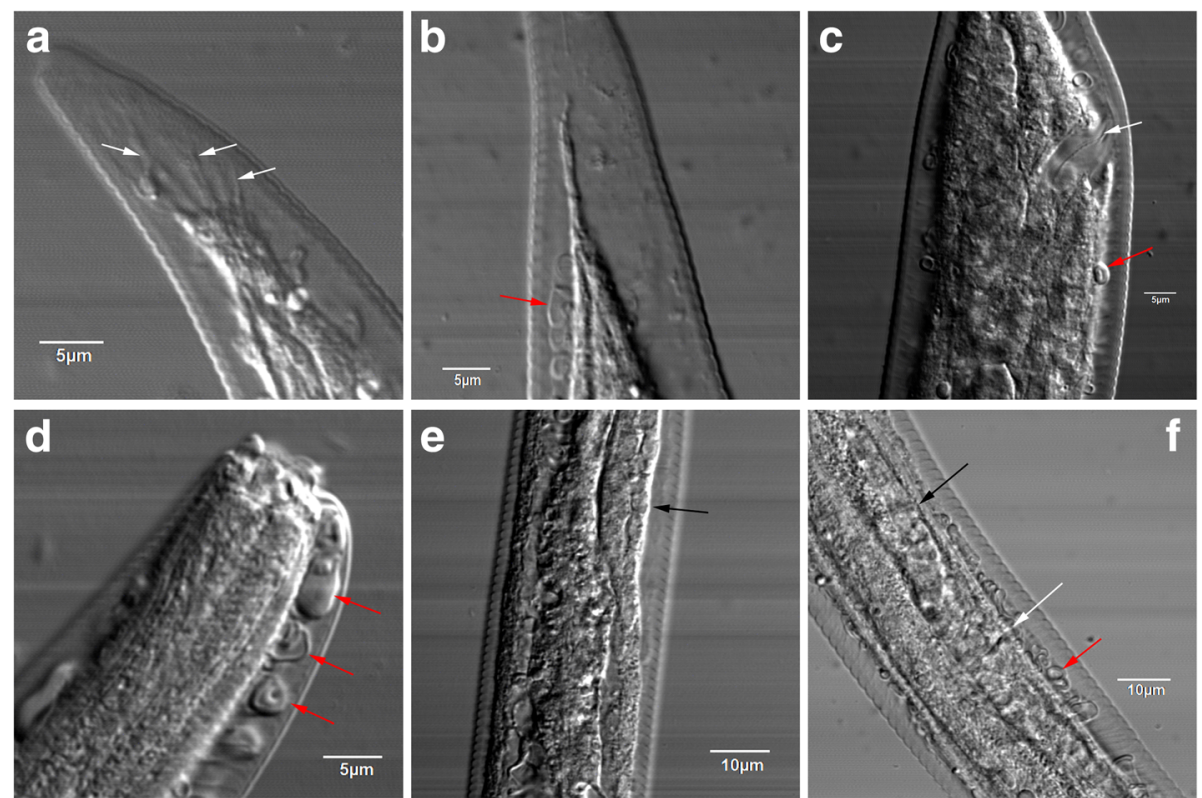

Fig. 1 Morphological features of Haemonchus contortus larvae collected from gerbils. a The vestigial copulatory bursa (white arrows) at the posterior extremity of a male larva 4 days post-infection (dpi). b Posterior extremity of a female larva 4 dpi. c Excretory pore (white arrow) at the rear end of intestine of a female larva 4 dpi. $\mathbf{d}$ Buccal capsule at the anterior end of a female larva 4 dpi. e Genital primordium (black arrow) of a female larva 4 dpi. $\mathbf{f}$ Genital primordium (black arrow) and vulva ovejector primordium (white arrow) of a female larva 7 dpi. The specific tissues below the cuticle are marked with red arrows in $\mathbf{b}, \mathbf{c}, \mathbf{d}$ and $\mathbf{f}$

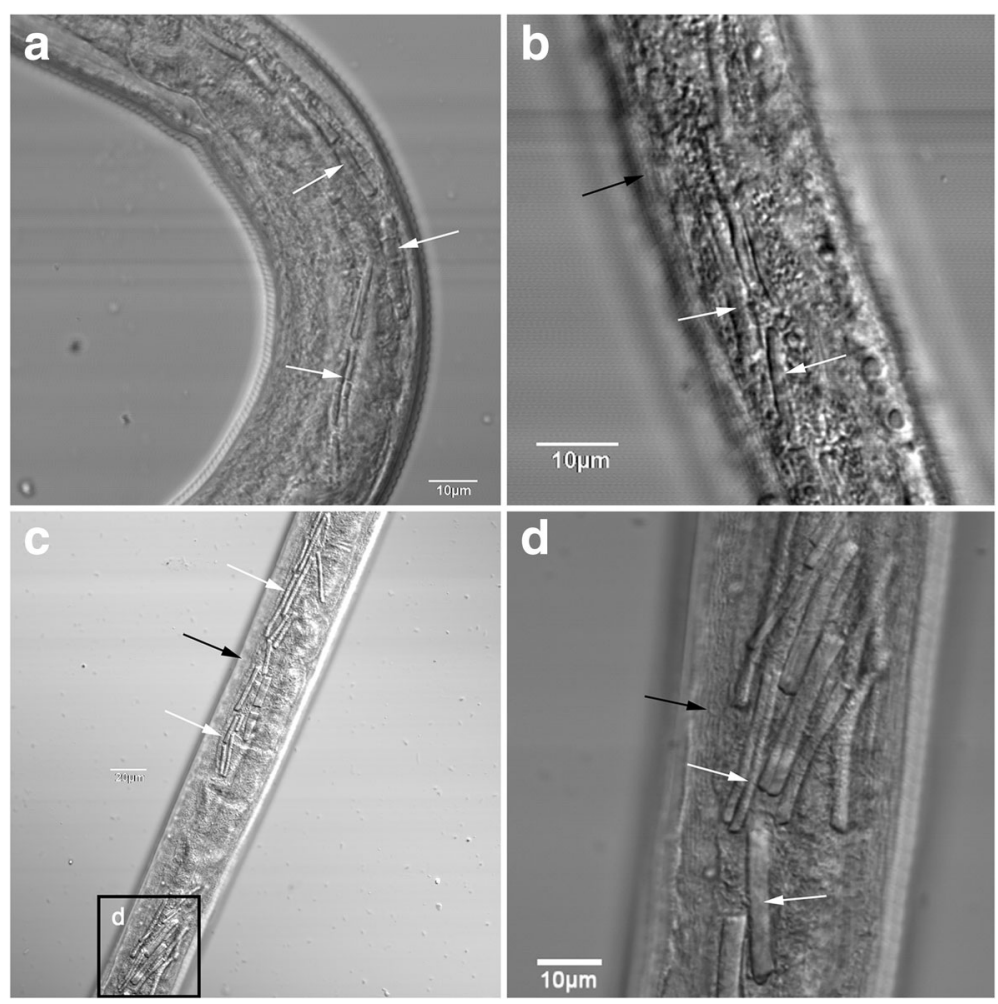

Fig. 2 Crystalline inclusions in the intestinal cells of Haemonchus contortus larvae. a Male larva 7 days post-infection (dpi). b Female larva 7 dpi. c, d Female larva $18 \mathrm{dpi}$. Crystalline inclusions are indicated by white arrows, and intestinal lumen is indicated by a black arrow 
found to increase with infection duration. In addition, the intestinal cells were also found to be solidly packed with crystals at $18 \mathrm{dpi}$ (Fig. 2d).

Upon histological examination, larvae were found, in the form of cross-sections with the intestinal lumen inside, in the gerbil lumens and mucosal surfaces of stomach (Fig. 3). The presence of eosinophil infiltrates and lymphocytic aggregates, or obvious inflammatory changes, was barely detected.

\section{Discussion}

Previous studies have made great efforts to search for a laboratory model to take the place of a ruminant model when conducting in vivo $H$. contortus infection experiments. Gerbils infected with $H$. contortus were first used as an anthelmintic screening model [1]. This model provided useful and credible information about hostparasite interactions. Rodent models, not just gerbil, including mouse, hamster, chinchilla and rabbit, could improve discovery efficiency, which means more work could be done prior to being completed in ruminants. Small laboratory animals offer convenience in feeding and handling, as well as lowering the research cost.

The amount of $H$. contortus recovered from gerbils can reach up to more than $30 \%$ of the inoculums [2]. However the larvae that survived in our experiment accounted for only a small percentage $(2 \%)$ of the inoculums. The factors causing this difference might be various; the inoculation dose of larvae is probably the most important one. Conder et al. found that inocula of 5002000 xL3s resulted in relatively constant worm recoveries, which kept $5-30 \%$ of the inoculums, and a dose of 1000 larvae was chosen for further work [2]. Squires et al. $[4,5]$ gave $600 \mathrm{xL} 3 \mathrm{~s}$ to gerbils and about $10 \%$ larvae recovered. But unlike the above two experiments, De Jesus-Gabino et al. [3] administered 4000 sheathed larvae to each gerbil, and the recovery rate was just 1.6$2.0 \%$. The relationship between infection dose and recovery rate seems to be unpredictable and occasional. Other factors, such as larval exsheathment method, sex and age of gerbils, the individual difference of host and environmental factors, were all other possible factors. Further study is required to enhance the recovery rate of H. contortus.

According to the life-cycle described by Veglia [13] (cf. Georgi \& Georgi [14]), once ingested by the appropriate host, a ruminant for $H$. contortus, the L3 exsheath and grow to early L4 at $\sim 4 \mathrm{dpi}$, and then L4 at $\sim 7$ dpi. Specialized development of the buccal capsule for blood-feeding and sexual differentiation is available at the fourth stage, following by gametogenesis in the adult stage. Female adults lay eggs at $\sim 18$ dpi, following copulation with males. The body length of larvae from L3 to adult could be $\sim 1 \mathrm{~mm}$ to $\sim 15-$ $18 \mathrm{~mm}$ (male) or to $\sim 25-30 \mathrm{~mm}$ (female). In this experiment, the basic tissue differentiation and body length of larvae in gerbils was similar to that of normal larvae in sheep at $4 \mathrm{dpi}$. However larvae recovered from gerbils at 7 and $18 \mathrm{dpi}$ were significantly shorter than those in sheep at the same time point [2]. This was probably induced by host differences, since the gastric conditions, including $\mathrm{pH}$, gas composition and content, of gerbil and sheep are very different [15-18]. Even the growth extent was much lower than that found by Conder et al. [2], which also described a gerbil model. Larvae grew to the length of $1100 \pm 169 \mu \mathrm{m}, 1273 \pm 122 \mu \mathrm{m}$ and $1553 \pm 133 \mu \mathrm{m}$ at 4, 7 and $18 \mathrm{dpi}$, respectively, in our study, but grew to $1320 \pm 333 \mu \mathrm{m}, 2055 \pm 290 \mu \mathrm{m} \quad$ (male) and $5415 \pm 3589 \mu \mathrm{m}$ (male) at 4, 7 and $21 \mathrm{dpi}$, respectively, in the study of Conder et al. [2]. This could be explained by a different parasite strain and housing environment.

Haemonchus contortus can undergo arrested development at the fourth stage in sheep abomasum when threatened by host or environment [19, 20]. Arrest also refers to diapause in $H$. contortus. Diapause is defined by two unique characteristics $[11,15]$. First, it is induced by environmental cues, often seasonal changes, and the arrest is temporarily irreversible. Secondly, the body length of diapausing larvae is short and uniform. Resumption

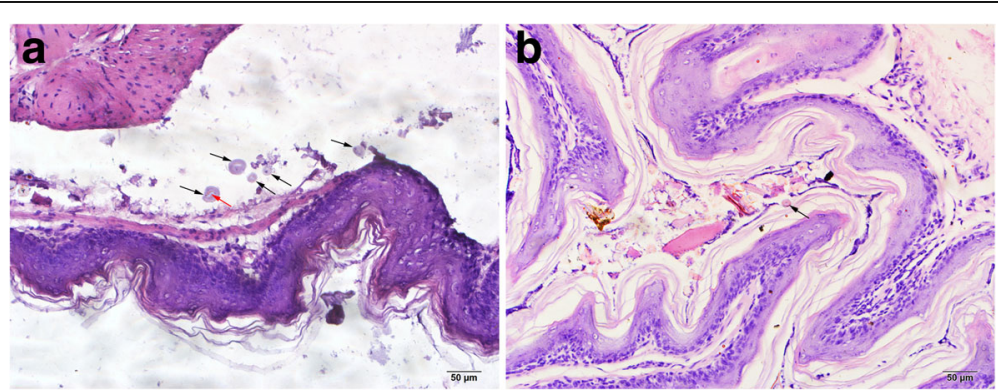

Fig. 3 Stomach sections of gerbils. a Larvae located in the lumen of stomach. $\mathbf{b}$ Larvae located at the mucosal surface of stomach. Cross-sections of early fourth-stage larvae at 4 days post-infection are marked with black arrows; the intestinal lumens of a larvae is marked with a red arrow in a 
of development will not proceed unless diapause development has been completed and environmental conditions are favorable. In $H$. contortus, diapause ensues when infective larvae (iL3s) undergo development for 3 or 4 days to reach the early fourth-stage after host infection [21]. Larvae recovered from gerbils in our experiment were not uniform, although they were short. The most important point was that larvae became longer with increased infection length. Thus, they just grew at a much slower speed in an inappropriate host, rather than enter into diapause.

Another symbolic feature of diapausing $H$. contortus is the presence of rod-like crystalline inclusions in the intestinal cells [21]. While these inclusions can still be found in the adult stage when the diapausing larvae have resumed development, they were not detected in normally developing larvae. In this study, the rod-like crystalline inclusions appeared in the intestinal cells at $7 \mathrm{dpi}$ and were present from this time on. This is a new discovery which was absent in the study of Conder et al. $[1,2]$. Earlier work has reported the presence of protein and sulphur in the crystals [15], while our recent DAPI staining results revealed positive nucleic acid staining [11]. Although the significance of these compounds remain unknown, it is possible that they were built up metabolic waste products generated during the process of development [21, 22]. Colglazier et al. [23] supported this theory, in which diapausing $H$. contortus conferred great resistance to most anthelmintics including thiabendazole. As such, it is reasonable that the number of crystals increased with infection days, as the metabolic rate and energy requirements were probably greatly reduced in slowly developing $H$. contortus. Rodlike crystalline inclusions were also present in $H$. contortus recovered from rabbits, but without any explanation to this phenomenon [8].

Since larvae were mostly located in the lumens or on the stomach mucosal surfaces, eosinophils or other inflammatory responses were not obvious in our results. We performed routine peripheral blood examination after gerbil infection, and found few changes in the numbers of red blood cells, eosinophils or lymphocytes (data not shown). This indicated that, unlike sheep, gerbils infected with $H$. contortus failed to establish a significant systemic immune response, or even a local reaction. It is possible that $H$. contortus were being regarded as food items and subsequently digested in non-routine hosts. Only the most adaptive larvae survived, but they failed to develop to adulthood. In addition, it is possible that the small number (about $2 \%$ of the inoculums in our study) of larvae was insufficient to elicit effective immune responses. Gerbils were immunosuppressed by dexamethasone, and therefore this may also affect their immune responses. A higher larval dose would be required to stimulate a local reaction [2].

\section{Conclusions}

The present study has successfully obtained fourth-stage $H$. contortus larvae in a gerbil infection model. Morphological characterization of the larvae revealed shorter larval length but almost the same appearance, which suggested that slower development, rather than arrested development, has taken place in gerbils, compared to in sheep. The presence of rod-like crystalline inclusions in nematode intestinal cells was found, indicating that slowly developing $H$. contortus had a reduced metabolic rate. Our results provide an additional description of the development of $H$. contortus ZJ strain from gerbils and may contribute to enhance the anthelmintic screening model.

\section{Abbreviations \\ ANOVA: Analysis of variance; dpi: Days post-infection; $\mathrm{HCl}$ : Hydrochloric acid; HE: Hematoxylin-eosin; iL3s: Infective larvae; L3: Third-stage larvae; L4: Fourth- stage larvae; SD: Standard deviation; XL3s: Exsheathed infective larvae; ZJ: Zhejiang}

\section{Acknowledgements}

We thank Zhejiang Academy of Agricultural Sciences for their assistance in collecting $H$. contortus eggs.

\section{Funding}

The project was supported by a grant from the National Key Basic Research Program (973 Program) of China (No. 2015CB150300), the National Key Research and Development Program of China (No. 2017YFD0501200) and the National Natural Science Foundation of China (No. 31602041, 31372426).

\section{Availability of data and materials}

The data supporting the conclusions of this article are included within the article.

\section{Authors' contributions}

DAF built and guided the team. YY designed the experiments. HY cultured the $H$. contortus iL3s from eggs, exsheathed iL3s, infected gerbils with larvae and killed gerbils. YY, ZHL and GXL collected larvae from stomachs, observed morphological features and took photomicrographs. CXQ made the stomach sections and took pictures. YY and GXL dealt with data and figs. YY wrote the manuscript. All authors read and approved the final manuscript.

\section{Ethics approval and consent to participate}

All animals were treated in strict accordance with the recommendations of the Guide for the regulation for the Administration of Affairs concerning Experimental Animal of the People's Republic of China. The experiments were approved by Zhejiang University Experimental Animals Ethics Committee (Permit Number: ZJU201308-1-10-072).

\section{Consent for publication}

Not applicable.

\section{Competing interests}

The authors declare that they have no competing interests.

\section{Publisher's Note}

Springer Nature remains neutral with regard to jurisdictional claims in published maps and institutional affiliations.

\section{Author details}

${ }^{1}$ Institute of Preventive Veterinary Medicine, Zhejiang Provincial Key Laboratory of Preventive Veterinary Medicine, College of Animal Sciences, Zhejiang University, Hangzhou 310058, China. ${ }^{2}$ Zhejiang Center for Animal Disease Control and Prevention, Hangzhou 310000, China. 
Received: 4 May 2017 Accepted: 11 October 2017

Published online: 23 October 2017

\section{References}

1. Conder GA, Jen LW, Marbury KS, Johnson SS, Guimond PM, Thomas EM, et al. A novel anthelmintic model utilizing jirds, Meriones unguiculatus, infected with Haemonchus contortus. J Parasitol. 1990;76:168-70.

2. Conder GA, Johnson SS, Hall AD, Fleming MW, Mills MD, Guimond PM. Growth and development of Haemonchus contortus in jirds, Meriones unguiculatus. J Parasitol. 1992;78:492-7.

3. De Jesus-Gabino AF, Mendoza-de Gives P, Salinas-Sanchez DO, Lopez-Arellano ME, Liebano-Hernandez E, Hernandez-Velazquez VM, et al. Anthelmintic effects of Prosopis laevigata $\mathrm{n}$-hexanic extract against Haemonchus contortus in artificially infected gerbils (Meriones unguiculatus). J Helminthol. 2010;84:71-5.

4. Squires JM, Ferreira JF, Lindsay DS, Zajac AM. Effects of artemisinin and Artemisia extracts on Haemonchus contortus in gerbils (Meriones unguiculatus). Vet Parasitol. 2011;175:103-8.

5. Squires JM, Foster JG, Lindsay DS, Caudell DL, Zajac AM. Efficacy of an orange oil emulsion as an anthelmintic against Haemonchus contortus in gerbils (Meriones unguiculatus) and in sheep. Vet Parasitol. 2010;172:95-9.

6. Sommerville RI. Development of Haemonchus contortus to the fourth stage in an abnormal host, the laboratory mouse. J Parasitol. 1977;63:952-4.

7. Ostlind DA, Mickle WG, Smith S, Ewanciw DV, Cifelli S. Efficacy of ivermectin versus dual infections of Haemonchus contortus and Heligmosomoides polygyrus in the mouse. J Parasitol. 2013;99:168-9.

8. Hutchinson GW, Slocombe JOD. Experimentally induced Haemonchus contortus infections in the rabbit. J Helminthol. 1976;50:143-52.

9. Wagland BM, Abeydeera LR, Rothwell TL, Ouwerkerk D. Experimental Haemonchus contortus infections in guinea pigs. Int J Parasitol. 1989;19: 301-5.

10. Boisvenue RJ, Hendrix JC. Susceptibility of Chinchilla spp. to Haemonchus contortus. J Parasitol. 1968:54:183-5.

11. Yan B, Guo X, Zhou Q, Yang Y, Chen X, Sun W, et al. Hc-fau, a novel gene regulating diapause in the nematode parasite Haemonchus contortus. Int J Parasitol. 2014:44:775-86.

12. Rothwell JT, Sangster NC. An in vitro assay utilising parasitic larval Haemonchus contortus to detect resistance to closantel and other anthelmintics. Int J Parasitol. 1993;23:573-8.

13. Veglia F. The anatomy and life-history of the Haemonchus contortus (Rud.). Rep Dir Vet Res. 1915;3-4:347-500.

14. Georgi JR, Georgi ME. Parasitology for veterinarians. 5th ed. Philadelphia: W. B. Saunders Company; 1990.

15. Sommerville RI, Davey KG. Diapause in parasitic nematodes: a review. Can J Zool. 2002;80:1817-40.

16. Stringfellow F. Cultivation of Haemonchus contortus (Nematoda: Trichostrongylidae) from infective larvae to the adult male and the egglaying female. J Parasitol. 1986;72:339-45.

17. Sommerville RI. The development of Haemonchus contortus to the fourth stage in vitro. J Parasitol. 1966;52:127-36.

18. Mapes CJ. The development of Haemonchus contortus in vitro. I. The effect of $\mathrm{pH}$ and $\mathrm{pCO}_{2}$ on the rate of development to the fourth-stage larva. Parasitology. 1969;59:215-31.

19. Guo X, Zhang H, Zheng X, Zhou Q, Yang Y, Chen X, et al. Structural and functional characterization of a novel gene, Hc-daf-22, from the strongylid nematode Haemonchus contortus. Parasit Vectors. 2016;9:422.

20. Ding $H$, Shi H, Shi $Y$, Guo $X$, Zheng $X$, Chen $X$, et al. Characterization and function analysis of a novel gene, $\mathrm{Hc}$-maoc-1, in the parasitic nematode Haemonochus contortus. Parasit Vectors. 2017;10:67

21. Blitz NM, Gibbs HC. Morphological characterization of the stage of arrested development of Haemonchus contortus in sheep. Can J Zool. 1971;49:991-5.

22. Martin JF, Lee DL. Observations on crystals found in the intestine of Nematodirus battus during the development of immunity to this nematode in lambs. Parasitology. 1975;72:75-80.

23. Colglazier ML, Kates KC, Enzie FD. Anthelmintic activity of tetramisole, thiabendazole, and purified fine particle phenothiazine against experimental infections of Haemonchus contortus and Trichostrongylus species in sheep. Proc Helm Soc Wash. 1969;36:68-74.

\section{Submit your next manuscript to BioMed Central and we will help you at every step:}

- We accept pre-submission inquiries

- Our selector tool helps you to find the most relevant journal

- We provide round the clock customer support

- Convenient online submission

- Thorough peer review

- Inclusion in PubMed and all major indexing services

- Maximum visibility for your research

Submit your manuscript at www.biomedcentral.com/submit
Biomed Central 